\title{
Irrenzählung und Gründung psychiatrischer Kliniken im 19. Jahrhundert
}

\author{
Bern als Wegbereiter für andere Schweizer Kantone \\ von Hans Rudolf Wilhelm
}

\section{ZuSAMMENFASSUNG}

Die Psychiatrie begann im letzten Jahrhundert auch in der Schweiz als eigenständiger Zweig der Medizin zu wachsen; in manchen Kantonen wurden geistig abnorme Menschen gezählt. Die verantwortlichen Ärzte wollten damit den Behörden den Bedarf an staatlichen Irrenhäusern aufzeigen und sie von der Notwendigkeit solcher Bauten überzeugen. Eine Pionierrolle spielte dabei die Ärzteschaft des Kantons Bern. Neben epidemiologischen Fakten zeigen die Erhebungen auch einen Konnex von seelischem Leiden und sozialer Wirklichkeit.

\section{Einleitung}

In seiner Denkschrift «Über das öffentliche Irrenwesen in der Schweiz» hat 1846 Johann Matthias Hungerbühler (1805-1884), St. Galler Regierungsrat und Präsident der Schweizerischen Gemeinnützigen Gesellschaft, den fast durchwegs deplorablen Zustand des Psychiatriewesens jener Zeit beschrieben, wenn überhaupt eines bestand.

«Privatkliniken für Irren [sic!], zumal zweckmässig eingerichtete und von bedeutenderm Umfange, befanden sich in unserm Vaterlande von jeher nur sehr wenige. Sie wurden meist nur von Solchen benutzt, die eigenes Vermögen besassen und den höhern Ständen der Gesellschaft angehörten. Von einer Oberaufsicht und Kontrole [sic!] des Staates über diese oft mehr auf den Erwerb als auf den Heilzweck gerichteten Privatanstalten war überall nicht die Rede. Misshandlung der Irren, unrechtmässige Aufnahmen usw. waren möglich, ohne dass die Polizei- und Sanitätsbehörden im Geringsten sich darum bekümmerten'.» 
Hungerbühler forderte deshalb die Kräfte des Staates zur Errichtung öffentlicher Irrenanstalten auf, wozu die vorgängige statistische Registrierung der Kranken nötig wäre.

Im Kanton Bern wurde in der ersten Hälfte des 19. Jahrhunderts begonnen, Irre zu zählen, um eine Bedarfsprognose für zu errichtende Anstaltsplätze zu erhalten. Die erste Statistik von 1839 fiel in die Zeit, in der auch St. Gallen (SG, 1838) und Neuenburg (NE, 1844) ihre geisteskranken Einwohner zählten. Die Berner Spezialerhebung von 1871, ausgearbeitet von Rudolf Friedrich Fetscherin (1829-1892), wurde zum Vorbild für die Zählungen der Kantone Appenzell Ausserrhoden (AR), Freiburg (FR), Graubünden (GR) und Luzern (LU). - Nicht alle Kantone bedienten sich jedoch der Irrenzählung zur Planung ihrer Anstalten.

Im 19. Jahrhundert wurden in weiten Teilen Europas und der Vereinigten Staaten von Amerika Irrenhäuser zur Heilung und Pflege von Geisteskranken gebaut, für Menschen, die endlich das Odium des dämonischen Besessenseins losgeworden waren.

Für die Deutschschweiz hatte in jener Gründerzeit der deutsche Psychiater Wilhelm Griesinger (1817-1868) eine richtungsweisende Bedeutung. In seinem 1845 erschienenen Werk «Die Pathologie und Therapie der psychischen Krankheiten» formulierte er ein nosologisches Schema, welches für kantonale Irrenzählungen zum Modell wurde ${ }^{2}$ :

\section{Primitive geistige Anomalien (heilbar)}

Schwermut

Tobsucht

Wahnsinn

2 Sekundäre geistige Anomalien (unheilbar)

Verrücktheit

Blödsinn

\section{Zum Phänomen Irrenanstalt bemerkte Griesinger :}

«Jede Anstalt ist nichts Anderes, als ein Hospital für Gehirnkranke; jede, ganz besonders aber die Heilanstalten, müssen durchaus den Charakter eines Krankenhauses, und nicht etwa den eines Besserungs-Institutes, einer Fabrik, oder gar eines Gefängnisses darbieten. Hiemit ist zugleich gesagt, dass die Anstalt durchaus unter ärztlicher Leitung stehe, dass also die Direction in den Händen des ersten Arztes sein muss, der mit einer gewissen Unumschränktheit alle sonstigen Kräfte zum Besten des Ganzen verwendet, aber auch, dass die Irrenärzte wirkliche Ärzte, und nicht etwa Moralisten, welche sich zugleich etwas 
mit Medicin beschäftigen, aber zu jeder Untersuchung ihrer Kranken der Beihülfe eines weiteren Arztes bedürfen, sein sollen ${ }^{3} . »$

\section{Irrenzählungen und Anstaltsgründungen im Kanton Bern}

\section{Zur Vorgeschichte}

Die früheste weltliche Urkunde über einen Geisteskranken auf bernischem Gebiet stammt vom 5. März 1353. Es handelt sich um einen Mann, der in einem Tobsuchtsanfall eine Frau erschlagen hat. Er wird ausgewiesen und verpflichtet, seine Gemeinde nie mehr zu betreten. Hin und wieder gibt es Hinweise auf Geisteskranke in Gefängnissen oder in für sie errichteten Taubhäuschen, Einzelzellen aus Bretterverschlägen. Es existieren auch, vor allem aus der Zeit nach den Burgunderkriegen im 15. Jahrhundert, Bettelbriefe, welche den Geisteskranken vom Schultheiss und dem Rat zu Bern ausgestellt wurden, und wo der Name des Kranken erwähnt ist. In diesen Schreiben wird allen Leuten, besonders aber den geistlichen und weltlichen Beamten, empfohlen, den Träger gut zu empfangen, ihn zu unterstützen und weiter gut zu empfehlen. Namentlich erwähnt wurden auch bis gegen Ende des 15. Jahrhunderts Selbsttöter. Ihre Leichen wurden dem Henker übergeben, verstümmelt, ins Wasser geworfen oder verbrannt, die Angehörigen mit schweren Bussen belegt. Misslang ein Suizidversuch, so wurde der Mensch gepflegt, bis er sich erholt hatte, dann wie ein Mörder hingerichtet. Ein Ratsmanuale von 1487 verbietet einem geisteskranken Mann den Eheschluss. Im Manuale von 1517 wird ein Kaufvertrag eines Geisteskranken für null und nichtig erklärt. In Taufrodeln aus dem 17. Jahrhundert sind uneheliche Kinder von Geisteskranken und deren Eltern, soweit bekannt, aufgeführt ${ }^{4}$.

1749 wurde das erste staatliche, eigens für die Versorgung von Geisteskranken gebaute Haus, das Tollhaus, eröffnet. Es war ein einstöckiger Blockbau mit 12 Einzelzellen auf dem Breitfeld hinter dem Siechenhaus vor der Stadt Bern; dieser heute noch bestehende Bau wird im Gebäudekomplex der Psychiatrischen Universitätsklinik Bern, der Waldau, «Althaus» genannt. Anfangs unterstand das Tollhaus dem Grossen Spital und wurde vom Siechenvogt verwaltet. 1766 wurde es dem Ausserkrankenhaus angegliedert. Betreut wurden die Kranken durch den Stadtphysikus, der einmal im Monat, oder so oft es die Umstände erforderten, die Patienten besuchte, sowie durch den Chirurgus des Ausserkrankenhauses. 


\section{Beginn systematischer Zählungen: die Volkszählungen}

Kantonale Erfassungen von geistig abnormen Menschen entwuchsen den Volkszählungen. In seiner Sitzung vom 10. November 1835 beschloss das Departement des Innern, 1836 eine Volkszählung durchzuführen ${ }^{5} .1836$ folgte die Sanitätskommission einem entsprechenden Vortrage des Departements, gleichzeitig mit der Volkszählung eine Anomalenstatistik zu führen, damit eine:

«Übersicht über die Zahl und Zustand von Taubstummen, Blinden und Cretinen gewonnen werde $^{6} . »$

Die Anweisungen, die Anomalen in Tabellen aufzunehmen, lauteten:

«Die Ausfüllung der gedruckten Tabellen soll nun durch die Unterstatthalter gemeinschaftlich mit den Pfarrern stattfinden. Zu dem Ende sollen in jeder Einwohnergemeinde betreffende Namensverzeichnisse aller Taubstummen, Blinden und Blödsinnigen durch die ernannten Volkszähler aufgenommen und solche dem Unterstatthalter zugestellt werden. Derselbe untersucht mit den Pfarrern, ob die gezeichneten Personen wirklich in die Classe der Taubstummen, Blinden oder Blödsinnigen gehören ${ }^{7} . »$

Gezählt wurden im ganzen Kanton 1306 Blödsinnige, 690 Männer und 616 Frauen; dies entsprach einer Prävalenz von $3,27 \% 0^{8}$.

Bei der nächsten kantonalen Volkszählung von 1846 wurden geistig abnorme Menschen in «Blödsinnige» und «Wahnsinnige» aufgeteilt. Als blödsinnig, das hiess von Geburt an geisteskrank, galten 2527, als wahnsinnig, unter einer später erworbenen Krankheit leidend, 565 Menschen. Die tiefsten Krankenbestände mit zwischen 1,95 und 2,70\% lagen in den Bezirken des Landesteiles Jura. Die höchsten Prävalenzen, von 10,29 bis 11,95\% waren verteilt in den Bezirken der Landesteile Mittelland und Oberland. Der Bezirk Bern, wo auch die Patienten des Tollhauses lebten, verzeichnete $8,26 \%$ Kranke ${ }^{9}$.

Die Eidgenössische Volkszählung von 1870 brachte ein Novum: zum ersten Mal figurierte in einer landesweiten Zählung die Rubrik «geisteskrank». Als Grund dafür wurde angeführt, dass es von grossem volkswirtschaftlichen Interesse wäre, auch diejenigen Personen kennenzulernen und zu zählen, die aus gesundheitlichen Gründen momentan unproduktiv wären. Der Umstand, dass diese Frage noch bei keiner eidgenössischen Volkszählung gestellt worden war, wäre kein Grund, davor zurückzuschrecken :

«Im Gegenteil könne es der Schweiz nur zur Ehre gereichen, wenn sie in einer so wichtigen nationalökonomischen Frage die Initiative ergreife ${ }^{10} . »$ 
Im Kanton Bern wurden 1929 Kantonsbürger als geisteskrank gezählt, 919 weiblichen und 1010 männlichen Geschlechts; dies entsprach einem Bestand von $3,80 \%$ aller Berner ${ }^{11}$.

Diese drei Volkszählungen wurden ohne Ärzte durch Volkszähler, Unterstatthalter, Statthalter und Pfarrer ausgeführt; so ist es evident, dass die Resultate bezüglich der Geisteskranken mit Vorbehalt zu betrachten sind. Dies sahen damals auch Berner Ärzte, und im Bestreben, das Irrenwesen qualitativ wie quantitativ auszuweiten, vor allem auch durch den Bau neuer Irrenhäuser, traten sie beherzt auf und organisierten Erhebungen, die sich, anders als die Volkszählungen, nur mit Geisteskranken beschäftigen sollten.

\section{Die Irrenzählung von 1839 und die Gründung der kantonalen Anstalt Waldau 1855}

a) Zur Situation des Tollhauses um 1835

Nach zwei baulichen Erweiterungen hatte das Berner Tollhaus Platz für etwa 40 Patienten. Regierungsrat Hungerbühler führte in seiner Denkschrift von 1846 bezüglich des öffentlichen Irrenwesens in der Schweiz zum Kanton Bern folgendes aus:

«Der Zustand der Bernischen Irrenanstalt ist unter den gegebenen Verhältnissen ein leidlicher. Doch entspricht die Anstalt den Forderungen der Zeit und Bern's grossen Mitteln nicht. Von einer systematischen Sönderung der Heilbaren und der Pfleglinge nach den Fortschritten der heutigen Psychiatrie ist hier nicht die Rede. Die lobenswerthen Bestrebungen des leitenden Irrenarztes (Dr. Lehmann) scheitern nicht selten an der unzweckmässigen Lokalität. Die Anstalt leidet fast an allen Gebrechen, die an den alten Institutionen kleben, welche Irrenhäuser mit Krankenhäuser verbinden. Der Kanton Bern bedarf einer, seinen finanziellen Kräften angemessenen und seinen übrigen humanen Bestrebungen würdigen, mit den Fortschritten in der Psychiatrie im Einklang stehenden neuen IrrenHeil- und Pflegeanstalt ${ }^{12} .$,

Rund zehn Jahre früher, im Sommer 1834, hatte eine Kommission der dem Departement des Innern des Kantons Bern unterstellten Sanitätsabteilung die Zustände im Tollhaus untersucht und hauptsächlich folgende Übelstände gerügt :

«Zuerst das kerkerartige Aussehen der traurigen, schmutzigen, von Gestank erfüllten Zellen; dann die unzweckmässige Kost der Irren; ferner den Mangel an Reinlichkeit und an zweckmässiger Beschäftigung der Patienten; den Mangel gemeinschaftlicher Speise- und Arbeitszimmer $[\ldots]^{13} . »$ 
b) Professor Tribolet und die Medicinisch-Chirurgische Gesellschaft Die erste Anregung zur Errichtung einer neuen Irrenanstalt für den Kanton Bern war die Vorstellung von Professor Johann Friedrich Albrecht Tribolet (1794-1871) an den Grossen Rat im Jahre 1836. Tribolet, seit 1835 Arzt am Äusseren Krankenhaus, kannte das Tollhaus nicht bloss aus Anschauung, sondern aus einer vierjährigen Tätigkeit daselbst. Die Vorstellung Tribolets hat dem Rat offenbar keinen Eindruck gemacht; denn in seinem Verhandlungsbericht findet sich kein Vermerk ${ }^{14}$. Im Jahr darauf, 1837, unterstützte die «Medicinisch-Chirurgische Gesellschaft des Cantons Bern» Tribolets Idee. Damit war ihr der Erfolg beschieden. Eine Kommission von Sachverständigen wurde auf Anordnung des Departements des Innern bestellt, sie bestand aus drei Ärzten. Sie verlangten eine Irrenzählung im ganzen Kanton, welche dann auch 1839 ausgeführt wurde ${ }^{15}$.

c) Der Kommissionsbericht zur Zählung

Im dritten Heft der «Bernerschen Viertel-Jahrschrift» von 1840 hat die Kommission die «Statistik der Irren im Kanton Bern» von 1839 drucken lassen.

Gesamthaft wurden 570 Kranke gezählt; unterschieden wurden folgende Kategorien :

Wahnsinnige (277)

Melancholische (171)

Blödsinnige (122)

Nur knapp ein Drittel war vermögend; die Kommission zog daraus den Schluss:

«Es ist daher anzunehmen, dass im Allgemeinen die Armuth mit Allem, was sie in ihrem Gefolge führt, zu Geisteskrankheiten und namentlich zum Wahnsinn prädisponirt ${ }^{16} . »$

Nach Auswertung aller Fakten resümierten die Sachverständigen:

«Es würde uns zu weit führen und läge ausser unsern Zweck, hier näher in den [sic!] Detail einzutreten, welche Ausdehnung eine neue Irrenanstalt haben sollte; und noch weniger wollen wir uns hier einlassen, wie eine solche einzurichten wäre. Indessen denken wir, der reichste Staat von ganz Europa, der Kanton Bern, werde nicht hinter denjenigen Ländern zurückbleiben wollen, welche bei wenigern Mitteln und weniger Irren, weitaus grössere und zum Theil sehr zweckmässig eingerichtete Irrenanstalten besitzen ${ }^{17} . »$

Am 9. Februar 1850 beschloss der Grosse Rat, eine neue Irren-Heil- und Pflegeanstalt zu errichten ${ }^{18}$. Am 29. Juni wurde entschieden, der neuen 
Anstalt den Namen «Waldau» zu geben. 1855, am 18. November, fand die feierliche Eröffnung statt ${ }^{19}$.

\section{Die Irrenzählung von 1871}

a) Grund der Zählung

Der Grund dafür, dass der Kanton Bern die Zahl der geistig Kranken in einer Spezialerhebung erneut ermitteln wollte, war die seit Jahren schwer lastende Überbelegung der Waldau. Fetscherin, Sekundärarzt in der Waldau, schreibt darüber im «Correspondenzblatt für Schweizer Ärzte» 1872:

«Die Waldau, die im Maximum bei ihrem Bau auf 230 Kranke berechnet war, wozu durch Wiedereröffnung des alten Irrenhauses und durch Erwerbung des Neuhausgutes 70 fernere Plätze für Unheilbare gekommen sind, beherbergt fast ununterbrochen 20-30 überzählige Patienten, 320-330, und sieht sich leider genöthigt, jede Woche manche, selbst frische Fälle, zurückzuweisen, die dann sehr oft der öffentlichen Sicherheit wegen in dem Gefängniss untergebracht werden müssen. Von den in der Waldau im Jahre 1871 verpflegten 425 Patienten sind meist aus diesem Grunde 35 im Gefängniss momentan untergebracht gewesen ${ }^{20} . \gg$

Fetscherin wurde von der Direktion des Innern beauftragt, eine ausführliche Statistik von Irren und Idioten zu erstellen. Die Ergebnisse, Schlüsse und Forderungen, die sich aus der Arbeit ergaben, hat er in einem ausführlichen Bericht der Berner Regierung vorgelegt. Nur ein paar markante Stellen sollen erwähnt werden.

b) Zur Krankheitseinteilung

Es muss den berufenen Psychiater Fetscherin geschmerzt haben zu sehen, dass in der Eidgenössischen Volkszählung von 1870 in nur einer Rubrik «geisteskrank» alles erfasst wurde, was als Phänomen der Psychopathologie galt. Für die Zählung von 1871 hielt er sich an das erwähnte Schema von Griesinger und fasste es einfacher, auch für Laien verständlich zusammen in :

«l. Angeborene, d.h. von Geburt oder seit den ersten Lebensjahren bestehende Geisteskrankheit (Idiotismus und Cretinismus);

2. Seither erworbene Geisteskrankheit ${ }^{21} . »$

Abgesehen vom wissenschaftlichen Aspekt war die Unterscheidung in angeborene und in erworbene Formen, in die Idiotie und in das Irresein, für die künftige Versorgung der Kranken von Bedeutung; Fetscherin äusserte sich dazu : 
«Werden also Idioten auch in Anstalten selten anders als Drohnen sich verhalten und namentlich nie geheilt werden, so sehen wir dagegen unter den Irren und selbst unter der grossen Zahl von Unheilbaren, an Verrückheit und Blödsinn Leidende viele, welche unter dem beruhigenden Einflusse des Anstaltslebens, oft zwar erst nach schweren Zeiten krankhafter Störung ihren früher erlernten Beruf aufnehmen. Wir sehen Schuster, Schneider, Tischler, Schmiede, Schlosser, Gärtner und ganz besonders viele Frauen ihre früher gewohnte Arbeit Jahr aus Jahr ein mit einer Emsigkeit betreiben, welche vielen Gesunden Ehre machen würde ${ }^{22} . »$

\section{c) Durchführung}

In einem Zirkularschreiben wurden 60 Ärzte gebeten, alle Kranken, auch wenn sie 1870 nicht registriert worden waren, in ihre Statistik aufzunehmen, namentlich auch Epileptiker, Menschen mit Dementia senilis und solche im Delirium tremens. Dieser nosologische Hinweis war bedeutend; denn erst wenige praktizierende Mediziner waren irrenärztlich ausgebildet und die psychiatrische Klinik im Rahmen des Medizinstudiums an der Universität Bern, 1861 gegründet, gerade zehn Jahre alt. Für die einzelnen Aufnahmen dienten vorgedruckte Zählblätter. Die Erhebung erkundete Geschlecht, Heimat, Wohnsitz, Alter, Beruf, Zivilstand, Konfession, Vermögensstellung, Verpflegungsart, Form der Krankheit, Zeitpunkt der Erkrankung, Krankheitsursache, frühere Klinikaufenthalte, Gemeingefährlichkeit der Kranken usw.

\section{d) Ergebnisse, Schlüsse und Forderungen}

Im Sommer 1871 wurden im Kanton Bern 2804 Kranke gezählt, 1512 Idioten und 1292 Irre. 592 Irre waren Männer, 700 Frauen. Die Frauen waren vermehrt manisch und melancholisch, die Männer litten mehr an Blödsinn. Das Irresein war in den Lebensjahren von 30-50 am häufigsten.

$41,4 \%$ der Irren und $24 \%$ der Idioten waren finanziell gut gestellt. $\mathrm{Zu}$ diesen Zahlen bemerkte Fetscherin:

«Es möge diese Thatsache namentlich Diejenigen belehren, die glauben, dass der Staat nur für die armen Irren zu sorgen habe, und die Sorge für die Bemittelten einzig ihren Familien zu überlassen sei. Ohne unverhältnismässige und oft sicher für ihre Familien erdrückende Opfer wird es unmöglich sein, diese Leute alle gehörig ausser den Staatsanstalten unterzubringen. Man vergesse nicht, dass die meisten Privatanstalten blos [sic!] für die Aufnahme von sehr Bemittelten eingerichtet sind, während unser Land eine sehr grosse Zahl von kleineren Vermögen zählt, die sich nur in normalen Verhältnissen zu den Bemittelten zählen können und bei eintretendem Missgeschick, wie Erkranken des Familienhauptes, nach längerer oder kürzerer Zeit in die Kategorie der Unbemittelten übergehen würden. Da diese 
Klasse aber am meisten mitträgt an die Lasten des Staatshaushaltes, so hat sie sicher auch Anspruch auf die Mitbenutzung der öffentlichen Anstalten, selbstverständlich gegen entsprechende Bezahlung eines höheren Pflegesatzes ${ }^{23}$.»

Als Fazit kann gesagt werden, dass mit dieser Zählung der Bedarf an weiteren staatlichen Anstalten nachgewiesen worden war; denn es war bemerkenswert, dass von 1292 Irren mehr als die Hälfte in Familien verpflegt wurden, die restlichen waren wohl in Anstalten untergebracht, aber nicht nur in Irrenkliniken, sondern auch in öffentlichen Armenhäusern wie in Hindelbank und in der Bärau. Die Idioten und Kretinen, die von Geburt an Kranken (1512), waren sowieso auf einen lebenslangen Pflegeplatz angewiesen. Die Waldau, das wiedereröffnete alte Tollhaus, das Neuhausgut boten aber nur 300 Plätze für einen geregelten Betrieb. Fetscherin schrieb in seinem Bericht an die Regierung:

«1. Die Irrenzählung im Kanton Bern von 1871 ergibt, dass die Versorgung der Geisteskranken in diesem Kanton eine durchaus ungenügende ist. Bei einer Zahl von 1300 Geisteskranken mit Ausschluss der Idioten findet sich eine einzige öffentliche Heil- und Pflegeanstalt mit 300 Plätzen, welche seit Jahren überzählig besetzt sind.

2. Die Irrenpflege ist aus Rücksicht auf die Gemeingefährlichkeit der Geisteskranken und auf die Humanität Sache des Staates.

3. Die Creirung einer eigentlichen Pflegeanstalt ist ein dringendes Bedürfnis, und zwar in Rücksicht auf die agricole Bevölkerung des Kantons in Verbindung mit landwirthschaftlichem Betrieb ${ }^{24} . »$

Im Jahre 1895 wurde in Münsingen eine neue kantonale Irrenheilanstalt eröffnet. Sie war in einem aus Block- und Pavillonsystem gemischten Stil gebaut und für 500 Kranke gedacht.

1898 wurde in der 1142 gegründeten Praemonstratenserabtei Bellelay, die 1797 aufgehoben und rund hundert Jahre später zu einer kantonalen Irrenpflegeanstalt eingerichtet worden war, der Betrieb aufgenommen.

Mehrere Kantone - Appenzell Ausserrhoden, Freiburg, Graubünden, Luzern - folgten dem Berner Beispiel, wie der Verf. in seiner nicht veröffentlichten Dissertation (Bern 1989) nachgewiesen hat. Im folgenden wird noch die Entwicklung in den Kantonen Neuenburg und St. Gallen beleuchtet, die unabhängig von den Berner Erfahrungen schon 1849 resp. 1847 ihre kantonalen Heil- und Pflegeanstalten schufen.

\section{Kanton Neuenburg}

Anfangs des 19. Jahrhunderts bestand in Neuenburg keine öffentliche Anstalt für Geisteskranke. Die Tätigkeit der Behörden richtete sich darauf, 
störende Kranke eingeschlossen, wenn nötig angebunden zu halten. So schrieb beispielsweise der Bürgermeister von Valangin 1842 an den Staatsrat, dass ein gewisser Mann wegen seiner Geisteskrankheit bis zur Stunde noch immer eingekerkert sei ${ }^{25}$.

Dieser Notbehelf mocht noch angehen bei einer akuten und vorübergehenden Krankheitsexazerbation, war aber untauglich bei problematischen chronischen Verläufen. Deshalb plazierte man Irre nach Bellevaux in Besançon oder nach Dôle im Jura, zwei französischen Asylen. Wegen der langen Wartezeiten und der beträchtlichen Kosten war dies jedoch keine Lösung auf Dauer. Die Regierung richtete das Amt eines «Médecin du Roi» ein Neuenburg war damals noch preussisches Hoheitsgebiet - der die Zustände in der Anstalt Bellevaux untersuchen sollte. Sein Urteil war deutlich: Bellevaux war eine Haftanstalt für die Verbüssung leichterer Delikte, ein Bettlerheim, dessen Krankenabteilung auch Geisteskranke aufnahm. Und der Bürgermeister von Le Locle schreibt 1840 über Bellevaux: Man sendet in dieses Etablissement Übeltäter, Prostituierte, verurteilte Schuldner ${ }^{26}$.

In dieser schwierigen Situation kam die Schenkung von Auguste de Meuron (1789-1852) zu Hilfe. De Meuron, Neuenburger, verliess nach einer Handelslehre seine Heimatstadt und gründete in Brasilien Tabakfabriken. 1837 aus gesundheitlichen Gründen wieder zurückgekehrt, stiftete er das finanzielle Fundament einer künftigen Klinik für Geisteskranke ${ }^{27}$.

1844 wurde eine Irrenzählung (une statistique des aliénés) durch den Staatsrat angeordnet. Der «Médecin du Roi» bestimmte in allen Bezirken die ausführenden Ärzte ${ }^{28}$. Die nosologische Einteilung geschah nach der Lehre von Jean-Etienne-Dominique Esquirol (1772-1840). Es wurden 233 Kranke gezählt, 108 Männer und 125 Frauen.

«Rien n'était plus propre que ce recensement à faire ressortir la nécessité d'un établissement spécial affecté au traitement des maladies mentales; puisqu'il nous faisait connaître d'une part, que 48 aliénés étaient réputés curables, et de l'autre, que 23 seulement se trouvaient renfermés dans des maisons de santé. Plus de la moitié, par conséquent, vivaient en dehors des conditions favorables au traitement, et sur ce nombre la presque totalité demeuraient privés de soins médicaux. Il est évident que cette proportion d'aliénés curables non isolés se fût singulièrement réduite, si le pays eût possédé un établissement convenable pour leur traitement ${ }^{29} ; »$

denn, so zitierte der Neuenburger Arzt François Louis Bovet (1812-1897) den berühmten französischen Alienisten Esquirol:

«Une maison d'aliénés est le plus puissant instrument de guérison de la folie: elle a une action physique et morale sur les malades ${ }^{30} . »$ 
Dieses «Instrument» konnte dank der Schenkung von de Meuron bereitgestellt werden. Als Standplatz für den Bau wurde Préfargier in der Gemeinde Marin gewählt. Der Grosse Rat sanktionierte am 18. August die Statuten der Klinik $^{31}$. Am 1. Januar 1849 wurde der Betrieb unter der Leitung von Bovet aufgenommen.

\section{Kanton St. Gallen}

«Inzwischen veranlassten gemeingefährliche und schreckhafte Auftritte zweier an s.g. Mord-Monomanie leidender Gemüthskranker am 28. September 1836 die St.Gallische Regierung zu dem Beschlusse, den Zustand des Irrenwesens im Kanton und die Gründung einer Kantonalirrenanstalt in nähere Erdauerung zu ziehen ${ }^{32} . »$

Dieses eindrückliche Bild zeichnete der erwähnte Regierungsrat Hungerbühler aus seinem Heimatkanton. «In nähere Erdauerung» wurde die Gründung eines staatlichen Irrenwesens dann auch gezogen. Am 25. August 1837 nämlich beschloss die Sanitätskommission, ein Namensverzeichnis aller im Kanton lebender Geisteskranken aufzunehmen. Zu diesem Zwecke sollten sämtliche Bezirksärzte die Verzeichnisse ausfüllen ${ }^{33}$. Das Register enthielt die Namen von 526 Kranken.

«In der Folge des Ergebnisses dieser Statistik wurde der Eifer im Kleinen Rath so gross, dass man unter Umgangnahme von einem Minderheitsantrag, nach welchem die Irrenanstalt in die Gebäulichkeiten des inzwischen säkularisierten Klosters Pfäfers verlegt werden sollte, ausschliesslich an den Grund- und Neubau einer St.Gallischen Irrenanstalt dachte. . ${ }^{34} . »$

Da der Kostenvoranschlag für einen Neubau jedoch auf das dreifache der angenommenen Summe zu stehen kam, befasste sich die Regierung wieder mit der Klosterumwandlung. Am 14. November 1845 beschloss der Grosse Rat, in den Räumlichkeiten der aufgehobenen Benediktinerabtei Pfäfers die Irrenanstalt einzurichten ${ }^{35}$. Im August 1847 konnte Heinrich David Ellinger (1817-1873) als Direktor seine Arbeit beginnen.

\section{Schlussbemerkung}

Kantonale Irrenzählungen waren ein taugliches Mittel, ein öffentliches Psychiatrieween zu schaffen. Es wurden Zahlen von Geisteskranken eruiert, um die Ausmasse künftiger Kliniken zu planen. Allerdings ergaben die 
Erhebungen ein statisches Bild. Eine Woche, einen Monat später schon konnte die Prävalenz höher sein. So kam es dazu, dass im Lauf der Jahre die Zahl der Kranken diejenigen der letzten Zählung überstieg - die Anstalten hatten das Problem der Überbelegung.

In sozialer Hinsicht entlasteten die Irrenanstalten - heute psychiatrische Kliniken genannt - das Armenwesen der Gemeinden, die für arme Geisteskranke zu sorgen hatten, und die meist hoffnungslos überforderten Familien, die kranke Angehörige bei sich zu Hause pflegten. Regierungsrat Hungerbühler bewies einen guten Weitblick, als er in seinem Schlusswort über das öffentliche Irrenwesen in der Schweiz ausführte:

\begin{abstract}
«Wie sollen die Gemeinden ohne Armenhäuser die armen Kranken gut versorgen, bei denen die Hülfe in einem Privathause entweder gar nicht oder nur mit enormen Kosten möglich ist, oder wo es sich um geistig Unheilbare, z. B. um tobsüchtige und gemeingefährliche Irren handelt, deren Verpflegung in Privatwohnungen nach den häuslichen Verhältnissen schlechterdings unzulässig erscheint? Wir antworten: Hier eben, aber auch nur hier sollen die vereinten Kräfte im Staate den Familien und Gemeinden in Unterstützung der natürlichen Armuth durch Gründung und Stiftung von Landesirrenanstalten zu Hülfe kommen. Was diese für zweckmässige, erfolgreiche Heilung und Pflege der Kranken leisten, kann ohnehin in Gemeindearmenhäusern gar nicht, jedenfalls nicht mit so mässigen Kosten erzielt werden ${ }^{36} . »$
\end{abstract}

Eröffnungsjahr, Standort, Patientenkapazität und Bauart der ersten kantonalen Irrenanstalt:

\begin{tabular}{|c|c|c|c|c|}
\hline SG & 1847 & Pfäfers & 100 & Bauten in der aufgehobenen Benediktinerabtei \\
\hline $\mathrm{NE}$ & 1849 & Marin & 100 & Blocksystem \\
\hline $\mathrm{BE}$ & 1855 & Bern & 230 & Blocksystem \\
\hline $\mathrm{LU}$ & 1873 & St. Urban & 200 & Bauten in der aufgehobenen Zisterzienserabtei \\
\hline FR & 1875 & Marsens & 200 & Blocksystem \\
\hline GR & 1892 & Chur & 166 & Blocksystem \\
\hline AR & 1908 & Herisau & 250 & Pavillonsystem \\
\hline
\end{tabular}


Synopsis im interkantonalen Vergleich

Prävalenzvergleich der gezählten Irren, ohne Idioten und Kretinen, zwischen den Kantonen nach der Zählung von:

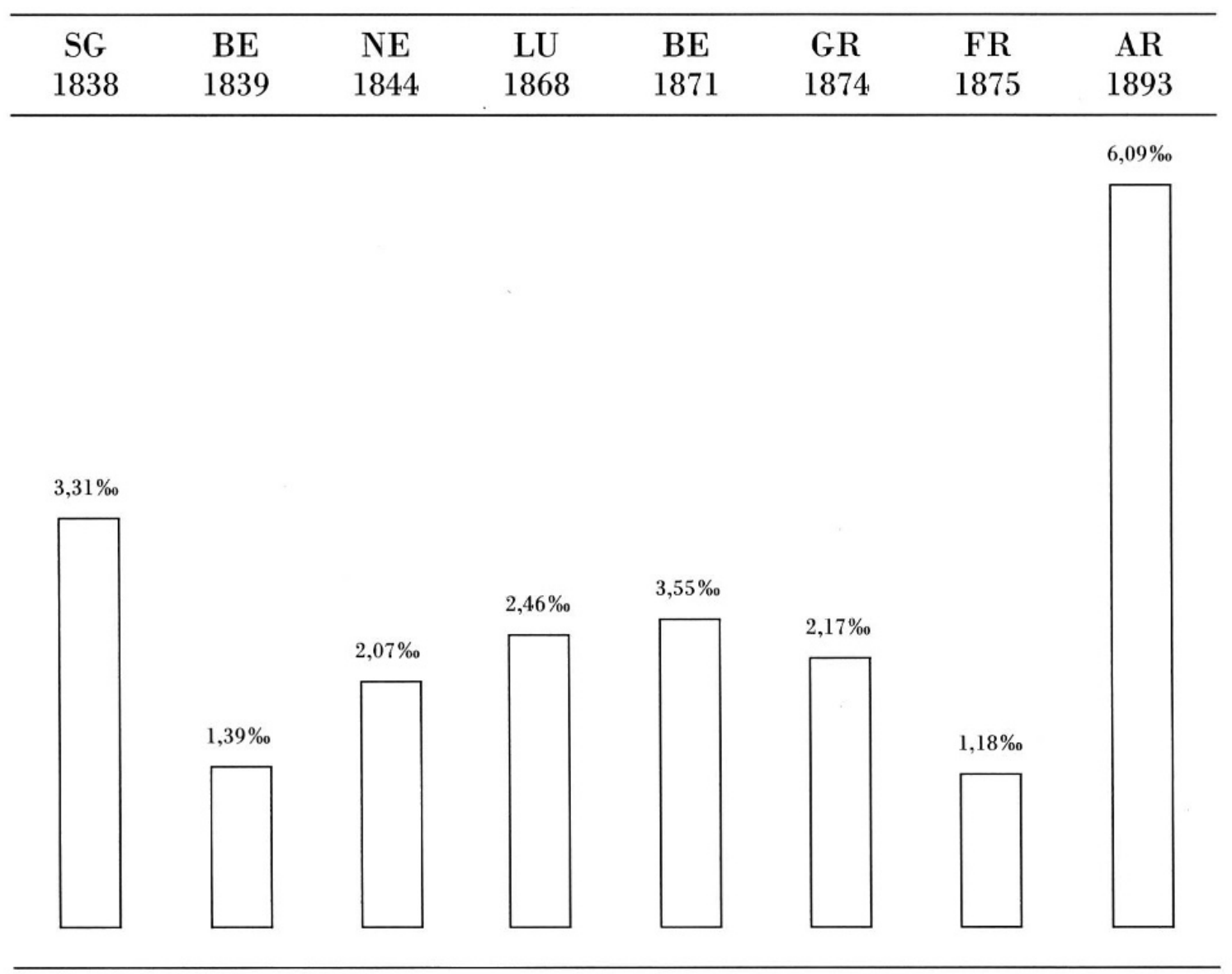




\section{Anmerkungen}

StA $=$ Staatsarchiv

1 Hungerbühler, J. M. : Über das öffentliche Irrenwesen in der Schweiz. St. Gallen und Bern 1846, S. 15-16.

2 Griesinger, W.: Die Pathologie und Therapie der psychischen Krankheiten. Stuttgart 1845, S. 152.

3 Ibid., S. 388.

4 Morgenthaler, W.: Bernisches Irrenwesen. Von den Anfängen bis zur Eröffnung des Tollhauses 1749. Bern 1915.

5 StA Bern: BB IV 20 Departement des Innern, S. 568.

6 StA Bern: BB XI 3 Sanitätsmanuale No. 106, S. 202

7 StA Bern: BB IV 21 Departement des Innern, S. 125-126.

8 StA Bern: Bericht des Regierungsrathes der Republik Bern an den Grossen Rath über die Staatsverwaltung in den Jahren 1836 und 1837, Beilage II.

9 StA Bern: Bericht des Regierungsrathes des Kantons Bern an den Grossen Rath über die Staatsverwaltung in den Jahren 1845-1848. Zweiter Bd., Tabelle Nr. 1 nach S.978.

10 Bericht der Conferenz zur Begutachtung der Vorlage des eidg. statistischen Bureau's über die Organisation der Volkszählung im Jahr 1870. Bern 1872, S. 3.

11 Schweizerische Statistik. Eidgenössische Volkszählung vom 1.Dezember 1870. 1. Bd. Hrsg. vom statistischen Bureau des Eidgenössischen Departement des Innern.

12 Cf. Anm. 1, S.34.

13 Imobersteg, J. : Das Inselbuch. Zweiter Theil, das äussere Krankenhaus, ehemals Siechenhaus. Bern 1878, S. 222.

14 Rechenschaftsbericht über die Verhältnisse und die Leistungen der verschiedenen Anstalten der Insel-Korporation von 1842 bis und mit 1873. Bern, S. 104.

15 Ibid.

16 Bernersche Viertel-Jahrschrift. 2. Bd., 3. Heft. Biel 1840, S. 58-88.

17 Ibid.

18 StA Bern: Gesetze, Dekrete und Verordnungen des Cantons Bern. Fünfter Bd. Jg. 1850, S. 24-27.

19 Cf. Anm. 13, S. 107-109.

20 Correspondenzblatt für Schweizer Ärzte, 2 (1872), 191.

21 Fetscherin, R. F. : Bericht an die Direction des Innern des Kantons Bern über die Zählung und Statistik der Geisteskranken und Idioten im Kanton Bern vom Jahre 1871. Bern 1872, S. 3 .

22 Ibid., S. 4.

23 Ibid., S.6-21.

24 Ibid.

25 StA Neuenburg: Série Police Sanitaire, Dossier 17/IV: Aliénés, Divers D.

26 StA Neuenburg: Série Police Sanitaire, Dossier 16/I : Aliénés, Généralités.

27 StA Neuenburg: Série Police Sanitaire, Dossier 16/V: Préfargier.

28 StA Neuenburg: MCE 206, Arrêt du Conseil D'Etat du 13 mai 1844.

29 Notice sur la maison de santé de Préfargier, Canton de Neuchâtel en Suisse. Par Louis Bovet, D. M. Neuchâtel 1848, S. 7. 
30 Ibid., S. 3.

31 StA Neuenburg: Bulletin officiel des délibérations du Grand-Conseil, 2 ${ }^{\circ}$ vol., p. 32-33.

32 Cf. Anm. 1, S. 53.

33 StA St. Gallen: R. 113 B 1, Protokolle der Sitzungen der Sanitätskommission in den Jahren 1837-1839, Artikel VIII, S. 74-75.

34 Cf. Anm.1, S. 53-54.

35 StA St. Gallen: R. 120 F. 5 a, Nr. 1: Organisation und Ordnung der St. Gallischen Heil- und Pflegeanstalt auf St. Pirminsberg in Pfäfers.

36 Cf. Anm.1, S. 67. 


\section{Summary}

Lunatic statistics and the foundation of psychiatric clinics in the $19^{\text {th }}$ centuryBerne as a pioneer for other Swiss Cantons

Psychiatry developed into an independant branch of medicine during the last century also in Switzerland. In most cantons statistics of the mentally abnormal were set up. In this way, responsible physicians proved to the authorities and to their fellow citizens the need for State lunatic asylums. In this respect, the medical practitioners in the Canton of Berne were pioneers.

\section{Résumé}

Recensement des aliénés et fondation de cliniques psychiatriques au $19^{e}$ siècleBerne comme pionnier pour d'autres Cantons suisses.

Au siècle dernier, la psychiatrie en tant que branche indépendante de la médecine s'est mise à prendre de plus en plus d'importance en Suisse aussi; dans la plupart des cantons, on a fait procéder à l'époque au recensement des presonnes anormales. Par ce moyen, les médecins responsables voulaient attirer l'attention des autorités sur les besoins existants en matière d'asiles d'aliénés publics et donc sur la nécessité de créer de tels établissements spéciaux. Les médecins du Canton de Berne furent appelés à jouer un rôle de pionniers dans ce contexte. Outre d'importantes données épidémiologiques, les sources des recensements effectués fournissent également un miroir de cette époque, où apparaît une relation étroite entre souffrances psychiques et réalité sociale.

Hans Rudolf Wilhelm, cand. med.

Winkelriedstrasse 25

CH-8006 Zürich 